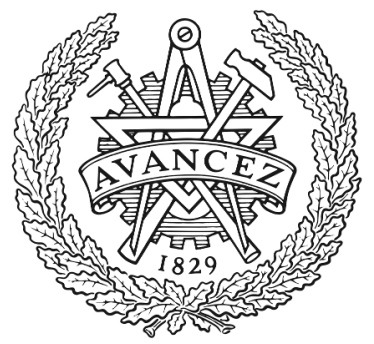

CHALMERS

UNIVERSITY OF TECHNOLOGY

\title{
Control of electric vehicles with autonomous corner modules: implementation aspects and fault handling
}

Downloaded from: https://research.chalmers.se, 2023-04-26 11:22 UTC

Citation for the original published paper (version of record):

Jonasson, M., Wallmark, O. (2008). Control of electric vehicles with autonomous corner modules: implementation aspects and fault handling. International Journal of Vehicle Systems Modelling and Testing, 3(3): 213-228. http://dx.doi.org/10.1504/IJVSMT.2008.023839

N.B. When citing this work, cite the original published paper. 


\title{
Control of electric vehicles with autonomous corner modules: implementation aspects and fault handling
}

\author{
Mats Jonasson* \\ Department of Chassis and Vehicle Dynamics, \\ Volvo Car Corporation, \\ SE-405 31 Göteborg, Sweden \\ E-mail: mjonass2@volvocars.com \\ ${ }^{*}$ Corresponding author
}

\section{Oskar Wallmark}

Swedish Center of Excellence in Electric Power Engineering, Department of Electrical Systems, Royal Institute of Technology, SE-100 44 Stockholm, Sweden E-mail: oskar.wallmark@ee.kth.se

\begin{abstract}
In this paper, vehicle dynamics for electric vehicles equipped with in-wheel motors and individual steering actuators are studied adopting the principles of optimal tyre-force allocation. A simple method for describing the constraints owing to tyre and actuator limitations is described. The control architecture is evaluated by investigating its response to realistic fault conditions. The evaluation demonstrates that the control architecture's ability to ensure vehicle stability generally is good. However, during major faults and extreme driving situations, vehicle stability is not maintained unless the constraints in the optimisation process used for tyre-force allocation are adapted to the specific fault.
\end{abstract}

Keywords: adhesion potential; ACM; autonomous corner module; electric in-wheel motor; electric vehicle; fault handling; force allocation; tyre constraints; vehicle control; vehicle dynamics.

Reference to this paper should be made as follows: Jonasson, M. and Wallmark, O. (2008) 'Control of electric vehicles with autonomous corner modules: implementation aspects and fault handling', Int. J. Vehicle Systems Modelling and Testing, Vol. 3, No. 3, pp.213-228.

Biographical notes: Mats Jonasson has been employed at Volvo Car Cooperation, Göteborg, Sweden, since 1998. His experience is in Design Engineering and he has an MSc in Electrical Engineering. At present, he works as an industrial $\mathrm{PhD}$ candidate in the Department of Chassis and Vehicle Dynamics at Volvo Car Corporation. His research covers vehicle dynamics on autonomous corner modules, in cooperation with the Department of Vehicle Dynamics, Royal Institute of Technology (KTH), Stockholm, Sweden.

Oskar Wallmark received the PhD from Chalmers University of Technology, Göteborg, Sweden, in 2006. He is currently working as a researcher within the Swedish Center of Excellence in Electric Power Engineering $\left(\mathrm{EKC}^{2}\right)$ at the Royal Institute of Technology (KTH) in Stockolm, Sweden. His research 
interests include control of electrical drives, particularly in hybrid and electric vehicle applications.

\section{Introduction}

One major trend in the automotive industry today is to replace mechanical and hydraulic actuators in vehicles with their electric counterparts. The justification for these changes is the added benefits of increased efficiency as well as comfort and safety related benefits (Chan and Wong, 2004; Emadi et al., 2006). To fit these parts into complex chassis and powertrain architectures, subsystems and components are also becoming modularised and distributed. This is enabled by the availability of electric power that can feed electric actuators locally, closer to which the generated force is needed (Zetterström, 2002; Dietrich et al., 2005; Siemens, 2006).

Adopting in-wheel (hub) motors combined with individual steering actuators enables the possibility of freely generating forces as closely as possible to the tyre contact patch. Furthermore, the tyre forces can be allocated (distributed) freely to each wheel. However, such vehicles are over-actuated (Valášek, 2003) and the tyre-force allocation problem can be represented by an under-determined set of equations. Over-actuation is tackled in Orend (2005) using a Moore-Penrose-inverse combined with an optimisation approach, which results in a tyre-force allocation where safety margins for tyre-force limitations are maintained at each wheel. A similar approach is adopted in Knobel et al. (2006) and evaluated on two different chassis configurations. The principle of optimal force allocation is generalised in Andreasson and Bünte (2006) so that it can be applied to a wide range of chassis configurations. Position and rate limitations of the actuators are also taken into account in the optimisation process.

Generally, if an optimisation approach is used to allocate the tyre forces, a significant computational capacity is required, which may render an on-board implementation difficult and expensive to realise. To reduce the number of computations, it is important that the optimisation problem is stated as simply as possible without losing the necessary accuracy in the solution proposed. Furthermore, the impact of non-ideal actuator and tyre dynamics, as well as actuator failures, on vehicle stability needs to be more thoroughly examined. These enquiries, reported in Andreasson et al. (2004) and Knobel et al. (2006), are approached here as described below.

\subsection{Contributions and outline of paper}

In this paper, vehicle dynamics are studied using the principles of optimal force allocation and closed-loop path control. A simple method for describing the constraints owing to tyre and actuator limitations is described thoroughly with an on-board implementation in mind. It is also shown how the adhesion potential of each tyre can be exploited by including a set of cost functions in the optimisation formulation.

The control architecture under consideration is evaluated by investigating its response to realistic fault conditions that can arise in electric vehicles equipped with in-wheel motors. First, the electric drivetrain is investigated, which consists of four in-wheel motors powered by a Lithium-ion battery combined with a supercapacitor. The system is 
simulated and its behaviour during a fault condition, referred to as an inverter shutdown (Jahns and Caliskan, 1999), is investigated. Second, the fault is injected and studied in a vehicle simulation model, which demonstrates that the control architecture under consideration is capable of handling these types of faults with only minor vehicle displacements. Third, a more potentially hazardous event, represented by the loss of power in one in-wheel motor and corresponding steering actuators, is also considered. It is shown that vehicle stability is not maintained unless the constraints used in the tyre-force allocation process are adapted to the specific fault. Finally, it is demonstrated how a limited computational capacity, manifested as slow sampling used in the optimisation solver, combined with the effect of non-ideal actuators, can result in unwanted interactions between the individual actuators.

The paper is outlined as follows. In Section 2, the vehicle specifications and model formulations are described. The approach for optimal tyre-force allocation is presented in Section 3. Finally, the results of the evaluation and conclusions are presented in Sections 4 and 5, respectively.

\section{Vehicle specifications and model formulations}

A mid-sized electric vehicle is considered in this paper. The total vehicle mass is $1400 \mathrm{~kg}$, of which $200 \mathrm{~kg}$ is attributed to a battery pack mounted on the body floor. Each vehicle corner is equipped with an in-wheel motor, individual steering actuators, friction brakes, and a spring/damper system. The vehicle is also equipped with an anti-roll bar. A modular concept providing all these functions is the Autonomous Corner Module (ACM) concept, described in Zetterström (2002) and Jonasson et al. (2006) and illustrated in Figure 1. An ACM prototype is currently developed and components to it will shortly be manufactured.

Figure 1 Top and side views of the ACM: (a) top view illustrating the steering actuators and suspension system (the damper is not shown) and (b) side view illustrating the integrated in-wheel motor and friction brake (see online version for colours)

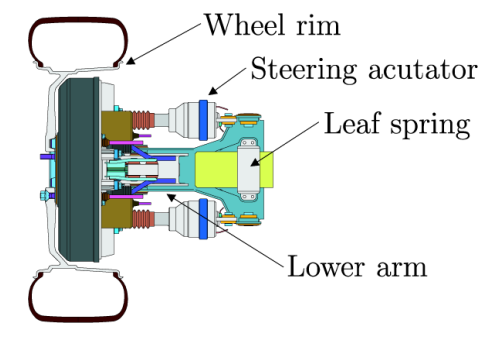

(a)

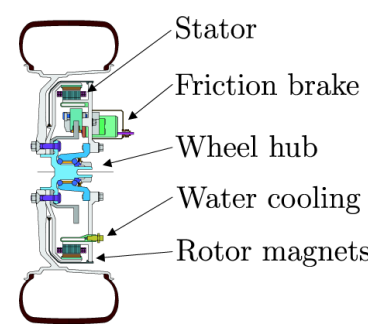

(b)

\subsection{Electric drivetrain}

The placement of components and schematics of the electric drivetrain (Bose, 2002) ${ }^{1}$ are shown in Figure 2. The permanent-magnet in-wheel motors are of an outer-rotor type and each can provide $15 \mathrm{~kW}$ of output power (used either to brake or to propel the vehicle). Each in-wheel motor is controlled by an inverter connected to a common DC side. The DC side consists of a lithium-ion battery connected in parallel with a DC/ 
DC-converter, which, in turn, is connected to a supercapacitor. By controlled switching of the transistors in the DC/DC-converter, the power demand from (or to) the in-wheel motors can be divided between the battery and supercapacitor. This enables the supercapacitor to function as a transient buffer that can provide (or absorb) power more rapidly than the battery whose dynamical response is much slower.

Figure 2 (a) Possible placement of electric machines, battery, supercapacitor and power electronics and (b) schematic of the electric drivetrain

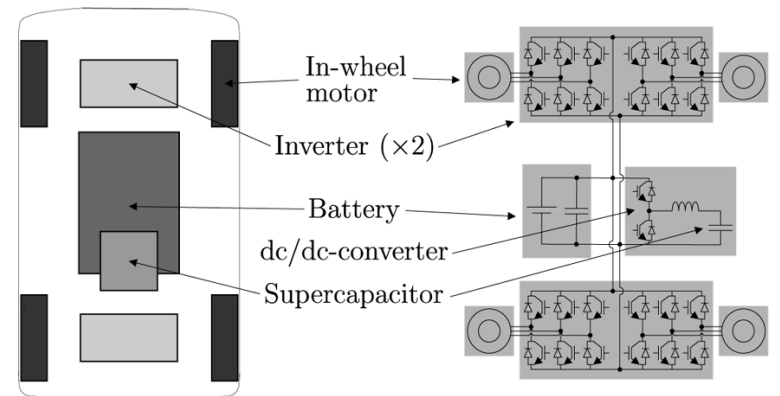

(a)

(b)

\subsection{Friction brakes and steering actuators}

Electromechanical friction brakes, where the brake pad in the caliper moves towards the brake disc guided by an electric actuator, are considered in this paper. The simple friction brake model adopted in this paper is shown in Figure 3. It is constituted by a saturation block, restricting the friction brake force within its limitations. The block is followed by a transfer delay block (it takes $50 \mathrm{~ms}$ for the brake pad to reach the disc). Finally, the pressure between pad and disk gradually increases, which is modelled as a first-order filter $(100 \mathrm{~ms})$. Thermal effects influencing the friction coefficient between pad and disc and thermal management of the electrical in-wheel motor are in this paper intentionally ignored. At low braking forces, all braking power is regenerated by the in-wheel motors and the major part can be used to charge the battery or supercapacitor. However, when the limit of maximum brake force is exceeded in the in-wheel motors, the friction brakes are activated so that the required brake force can be achieved.

Figure 3 Simple friction brake model and the principle of brake blending (see Table 1 in Appendix for a nomenclature list)

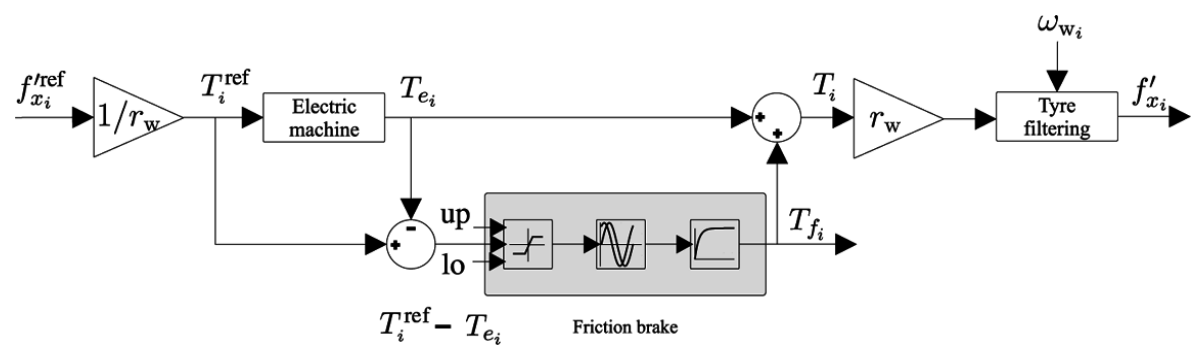

The steering actuators (see Figure 1(a)) move symmetrically around the vertical axis of the wheel and can provide steering angles within the interval $\pm 22^{\circ}$. The internal 
dynamics of the steering actuators are modelled as a rate limit of $\pm 1 \mathrm{rad} / \mathrm{s}$ combined with a first-order filter (100 ms).

\subsection{Closed-loop vehicle control}

The well-known two-track vehicle model governs the longitudinal $\left(v_{x}\right)$, lateral $\left(v_{y}\right)$ and yaw $\left(w_{z}\right)$ speeds of the vehicle. In addition, the effects of pitch, roll and bounce dynamics are included to account for the transient distribution of normal tyre forces during driving. Thus, the body of the vehicle has six degrees of freedom.

The vehicle movement follows a plane path defined by $v, \beta$, and $\rho$, where $v$ represents the vehicle's speed, $\beta$ the vehicle slip angle and $\rho$ the curvature of the path. A closed-loop path controller, further described in Jonasson and Wallmark (2006), is adopted to minimise the control error. The path controller outputs global force references, $\mathbf{f}_{\text {glob }}^{\text {ref }}=\left[\begin{array}{lll}F_{x}^{\text {ref }} & F_{y}^{\text {ref }} & M_{z}^{\text {ref }}\end{array}\right]^{T}$, acting at the vehicle's centre of gravity. The global forces are, in turn, allocated to each wheel corner as described in Section 3. The global, corner and tyre forces are illustrated in Figure 4, and a flow chart of the closed-loop vehicle controller is shown in Figure 5.

Figure 4 Global and corner forces acting on the vehicle. The tyre forces for the front-left wheel are also shown (see Table 1 in Appendix for a nomenclature list)

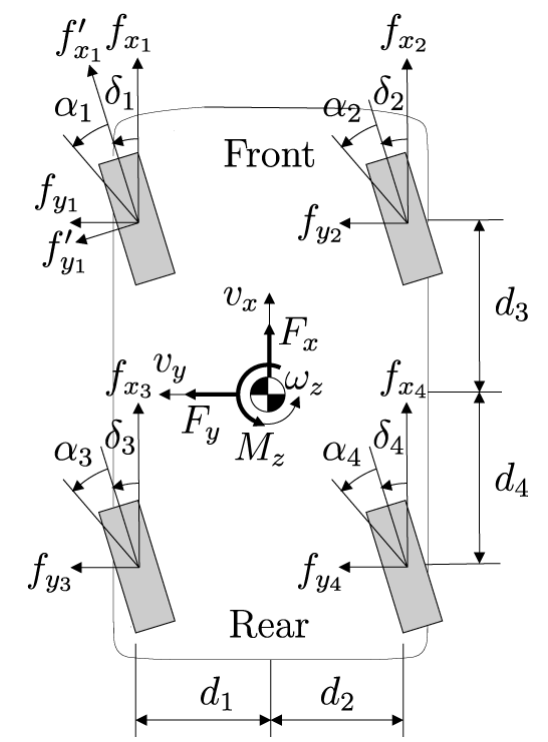

Figure 5 Flow chart of the closed-loop vehicle controller

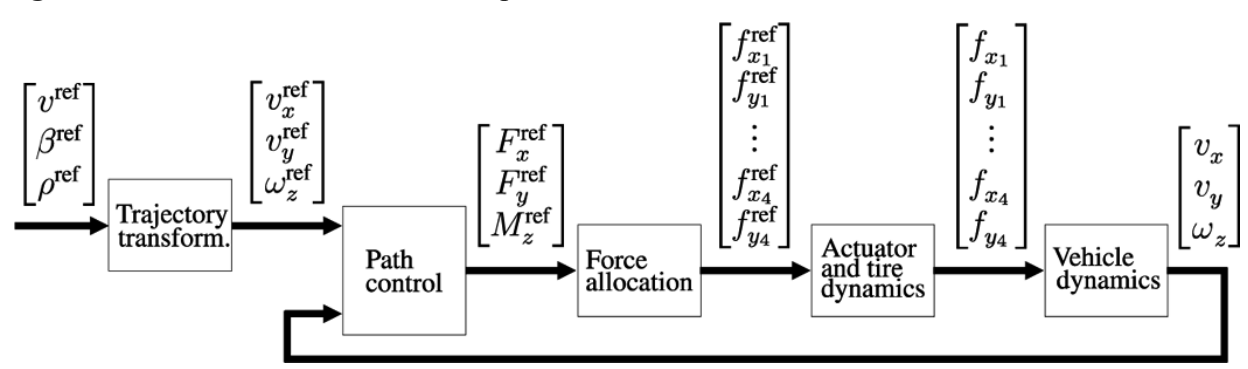




\section{A practical approach for tyre-force allocation}

\subsection{Constraints}

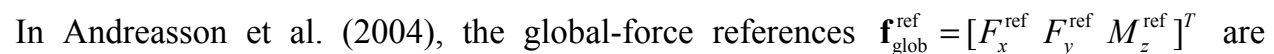
allocated as individual tyre-force references by solving an optimisation problem, taking both tyre and actuator constraints into consideration. In an on-board implementation, the number of computations is limited and the constrained optimisation problem should, therefore, be stated as simply as possible without losing the necessary accuracy in the solution proposed. A practical approach for how this can be realised as described below.

The limitations on longitudinal and lateral tyre forces on the $i$ th tyre are commonly described by the well-known friction ellipse. Figure 6(a) illustrates the limitations on tyre forces on a 225/50R 17-tyre obtained using a full-set magic tyre formula (Pacejka, $2002)$ at $3.8 \mathrm{kN}$ of normal load. Since the actuators may put additional restrictions on $f_{x_{i}}^{\prime}$ and $f_{y_{i}}^{\prime}$, the limitations applied by both the tyres and actuators can be formulated as

$$
\sqrt{\left(f_{x_{i}}^{\prime}\right)^{2}+\left(f_{y_{i}}^{\prime}\right)^{2}} \leq f_{i}^{\max }\left(\theta, \mu_{i}, f_{z_{i}}, \ldots\right),
$$

where $f_{i}^{\max }\left(\theta, \mu_{i}, f_{z_{i}}, \ldots\right)$ is the maximum available tyre force at the angle $\theta=\arctan \left(f_{y_{i}}^{\prime} / f_{x_{i}}^{\prime}\right)$. The boundary region dictated by equation (1) can be approximated using $n$ lines as illustrated in Figure 6(b) where approximations using $n=4$ and $n=8$ are shown. When adopting this approximation for all four tyres, the constraints owing to tyre and actuator limitations can be expressed as

$$
a_{1, i j} f_{x_{i}}^{\prime}+a_{2, i j} f_{y_{i}}^{\prime} \leq b_{i j}, \quad i=1,2,3,4 \quad j=1,2, \ldots, n,
$$

where $-a_{1, i j} / a_{2, i j}$ represents the gradient of the boundary and $\mathrm{b}$ is related to $f_{i}^{\max }$. By introducing $\mathbf{f}^{\prime}=\left[\begin{array}{llllllll}f_{x 1}^{\prime} & f_{y 1}^{\prime} & f_{x 2}^{\prime} & f_{y 2}^{\prime} & f_{x 3}^{\prime} & f_{y 3}^{\prime} & f_{x 4}^{\prime} & f_{y 4}^{\prime}\end{array}\right]^{T}$, equation (2) can be re-expressed in matrix form as

$$
\mathbf{A f}^{\prime} \leq \mathbf{b} \text {. }
$$

Equation (3) is expressed in terms of tyre forces. The tyre forces, in turn, are related to the corner forces as

$$
f_{x_{i}}^{\prime}=\cos \delta_{i} f_{x_{i}}+\sin \delta_{i} f_{y_{i}}, \quad f_{y_{i}}^{\prime}=\sin \delta_{i} f_{x_{i}}+\cos \delta_{i} f_{y_{i}}, \quad i=1,2,3,4
$$

where $\delta_{i}$ is the steering angle for the $i$ th tyre. By compiling the corner forces into a vector

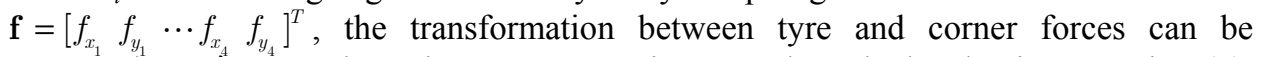
expressed as $\mathbf{f}^{\prime \prime}=\mathbf{T f}$, where the components in $\mathbf{T}$ can be calculated using equation (4). Hence, equation (3) can be re-expressed in terms of corner forces as

$\operatorname{ATf} \leq \mathbf{b}$.

The following simple example illustrates how equation (5) can be implemented. For the purpose of illustration, actuator limitations are intentionally ignored and only tyre-force limitations are taken into consideration. Furthermore, it is assumed that $f_{i}^{\max }$ is dependent only on vertical load and friction coefficient as 


$$
f_{i}^{\max }\left(\mu_{i}, f_{z_{i}}\right)=\mu_{i} f_{z_{i}}\left(k_{z_{1}}-k_{z_{2}} \frac{f_{z_{i}}-f_{z, \text { nom }}}{f_{z, \text { nom }}}\right),
$$

where $k_{z_{1}}, k_{z_{2}}$ and $f_{z, \text { nom }}$ are tyre parameters. Here, the influence of camber variations is neglected. Hence, $f_{x_{i}}^{\prime}$ and $f_{y_{i}}^{\prime}$ are limited within a circle which, in this example, is approximated using $n=4$ lines, forming the square shown in Figure 6(b). Using simple arguments of geometry, the constraints, in terms of tyre forces, on the $i$ th tyre are thereby approximated as

$$
\left[\begin{array}{cc}
1 & 1 \\
-1 & 1 \\
1 & 1 \\
-1 & 1
\end{array}\right]\left[\begin{array}{l}
f_{x_{i}}^{\prime} \\
f_{y_{i}}^{\prime}
\end{array}\right] \leq f_{i}^{\max }\left(\mu_{i}, f_{z_{i}}\right)\left[\begin{array}{cccc}
1 & 1 & -1 & -1
\end{array}\right]^{T}
$$

Figure 6 (a) Predicted tyre-force limitations from a full-set magic tyre formula on a 225/50R17-tyre and (b) linear piecewise approximation with $n=4$ and $n=8$

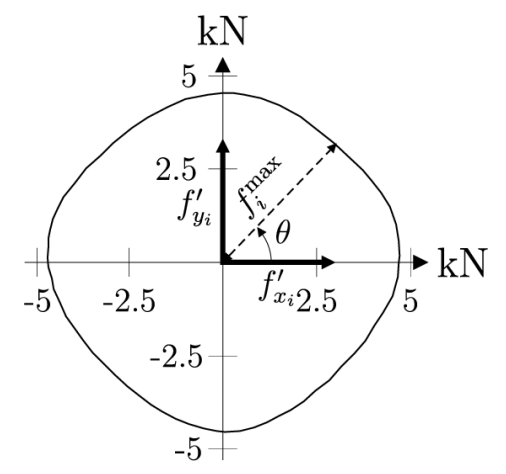

(a)

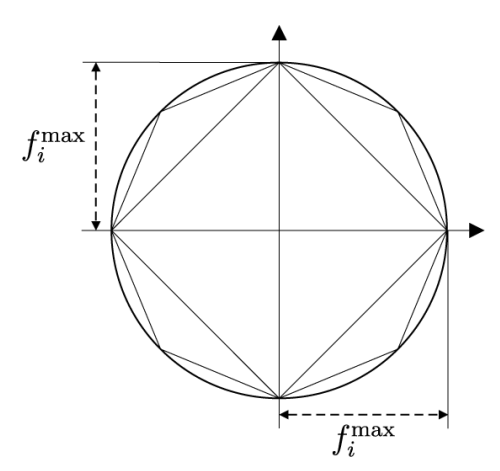

(b)

\subsection{Tyre-force allocation}

As discussed in Section 2.3, the outputs of the path controller are global force references, $\mathbf{f}_{\mathrm{glob}}^{\text {ref }}=\left[F_{x}^{\text {ref }} F_{y}^{\text {ref }} M_{z}^{\text {ref }}\right]^{T}$, that act at the vehicle's centre of gravity. The global forces are, in turn, related to the individual corner force references, $\mathbf{f}^{\text {ref }}=\left[f_{x_{1}}^{\text {ref }} f_{y_{1}}^{\text {ref }} \cdots f_{x_{4}}^{\text {ref }} f_{y_{4}}^{\text {ref }}\right]^{T}$, as

$$
\mathbf{f}_{\text {glob }}^{\text {ref }}=\mathbf{D} \mathbf{f}^{\text {ref }} \text {, }
$$

where

$$
D=\left[\begin{array}{cccccccc}
1 & 0 & 1 & 0 & 1 & 0 & 1 & 0 \\
0 & 1 & 0 & 1 & 0 & 1 & 0 & 1 \\
-d_{1} & d_{3} & d_{2} & d_{3} & -d_{1} & -d_{4} & d_{2} & -d_{4}
\end{array}\right]
$$


and $d_{1}, d_{2}, d_{3}$ and $d_{4}$ represent vehicle dimensions (see Figure 4). Equation (8) is under-determined and an infinite set of solutions exists (in terms of corner forces).

By adopting optimal allocation, the longitudinal $\left(f_{x_{i}}^{\prime}\right.$ ref $)$ and lateral $\left(f_{y_{i}}^{\prime}\right.$ ref $)$ tyre-force references can be distributed so that both equations (8) and (5) hold, i.e., the global forces required are attained taking into account the constraints put by tyres and actuators. However, it is desirable that the proposed solution also fulfils the following demands imposed. Tyre forces of high magnitude should generally be avoided to minimise tyre wear. In addition, since the vertical load on each tyre varies, the allocation of forces to each tyre should be adapted so that their full adhesion potential can be exploited, i.e., the proposed solution must be able to take advantage of an extension of tyre-force limitations arising from, e.g., an increase in friction coefficient or normal load.

The tyre and actuator constraints combined with the additional demands clarified above, are, in this paper, fulfilled by solving the following constrained, linear, least-square optimisation problem

$$
\min _{\mathbf{f}^{\text {ref }}} \frac{1}{2}\left\|\mathbf{W}\left[\begin{array}{c}
\mathbf{D} \\
\mathbf{I}
\end{array}\right] \mathbf{f}^{\text {ref }}-\mathbf{W}\left[\begin{array}{c}
\mathbf{f}_{\text {glob }}^{\text {ref }} \\
\mathbf{f}_{\text {cost }}
\end{array}\right]\right\|_{2}^{2}, \quad \mathbf{A} \mathbf{T} \mathbf{f}^{\text {ref }} \leq \mathbf{b} .
$$

In equation (10), $\mathbf{f}_{\text {cost }}$ represents cost functions added to fulfil the extra demands (the weight matrix, $\mathbf{W}=\operatorname{diag}\left(w_{1}, w_{1}, \ldots\right)$, is added to prioritise each row in the optimisation formulation). The proposed expression for $\mathbf{f}_{\text {cost }}$ is

$$
\mathbf{f}_{\cos t}=\frac{1}{4 m g \mu_{\Sigma}}\left[\mu_{1} f_{z_{1}} F_{x}^{\mathrm{ref}} \mu_{1} f_{z_{1}} F_{y}^{\mathrm{ref}} \cdots \mu_{4} f_{z_{4}} F_{x}^{\mathrm{ref}} \mu_{4} f_{z_{4}} F_{y}^{\mathrm{ref}}\right]^{T}, \quad \mu_{\Sigma}=\sum_{i=1}^{4} \mu_{i} .
$$

When $\mathbf{f}_{\text {cost }}$ is added to equation (10), the individual tyre forces generally divide the desired global force (references) evenly with the addition that the adhesion potential is exploited. This is accomplished in equation (11) by equalising the longitudinal and lateral corner forces by the vertical load and friction coefficient present $\left(\mu_{i} f_{z_{i}} / 4 m g \mu_{\Sigma}\right)$. The evenly distribution of $F_{x}^{\text {ref }}$ and $F_{y}^{\text {ref }}$ is used to avoid unacceptable high magnitudes of corner forces and to favour similar force interventions for all four corners. A flow chart illustrating the general principles of how the tyre forces are allocated is shown in Figure 7.

By using the steady-state inverse tyre model applied in Jonasson and Wallmark (2006) together with the actual wheel geometry, the resulting reference steering angles $\left(\delta_{i}^{\text {ref }}\right)$ can be computed. However, the electrical faults under consideration will introduce a rapidly growing wheel torque. To account for the transient response of the resulting longitudinal tyre force, the first-order tyre dynamics is added to the in-wheel motor and brake dynamics in Figure 7. This is also seen in Figure 3, where the longitudinal tyre dynamics is implemented as a speed depending filter. The computation of lateral tyre force relies on steady-state tyre model only, since steering in Section 4 will be smoothly aligned. 
Figure 7 Flow chart of the tyre-force allocation mechanism involving actuator dynamics (see Table 1 in Appendix for a nomenclature list)

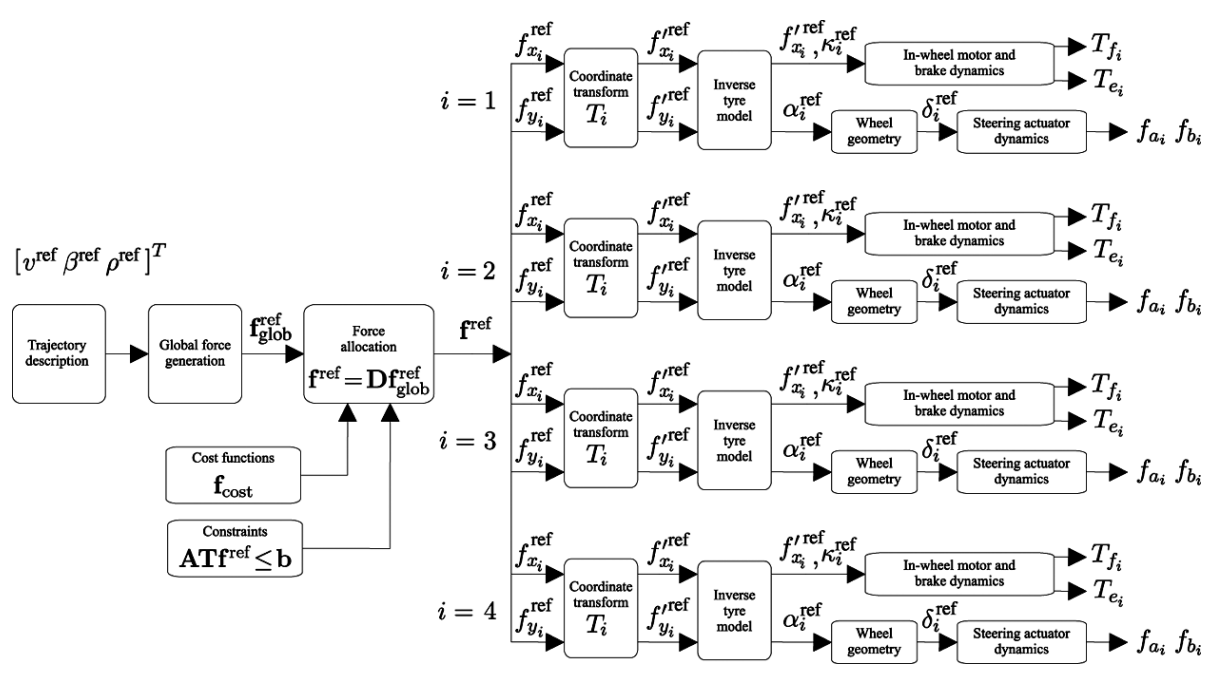

\section{Evaluation examples}

The control architecture under consideration is now evaluated by investigating its response to realistic fault conditions. For simplicity, it is assumed that the friction coefficient of each tyre is 0.95 . The constraints in the optimisation process are approximated as described in Section 3.1 using $n=8$ lines. Key parameters of the vehicle are reported in Table 2 in Appendix.

\subsection{Vehicle response to an inverter shutdown}

The electric drivetrain shown in Figure 2(b) has been simulated using MATLAB/Simulink ${ }^{2}$ and the power electronics simulation software PLECS. ${ }^{3}$ A Lithium-ion battery model, similar to the capacitor-network models presented in Gao et al. (2002) and Capel (2001), is adopted. The total capacitance of the supercapacitor is $20 \mathrm{~F}$ and the resistive and on-stage voltage drops in the transistors used in the inverters and DC/DC-converter correspond to Mitsubishi PM600DSA060 power transistor modules. The in-wheel motors adopt parameters (verified experimentally) from an experimental permanent-magnet in-wheel motor of outer-rotor type. More detailed descriptions of the electric drivetrain can be found in Jonasson and Wallmark (2006) and Wallmark (2006).

Figures 8(a)-(c) show a PLECS simulation of the electric drivetrain where the in-wheel motors are operating at a constant speed of $90 \mathrm{rad} / \mathrm{s}$, which corresponds to approximately $105 \mathrm{~km} / \mathrm{h}$ if a tyre radius of $r_{\mathrm{w}}=0.32 \mathrm{~m}$ is assumed and longitudinal tyre slip is ignored. In this simulation, all four in-wheel motors deliver a total of $30 \mathrm{~kW}$, which are evenly distributed to each wheel. At $t=5 \mathrm{~ms}$, the control signals to the inverter controlling the rear-left in-wheel motor, for some reason, vanish. This is referred to as an inverter shutdown (Jahns and Caliskan, 1999) and reasons for the origin of such a fault include failure of the control-supply voltage that powers the inverter, a local failure in 
the gate drive (supplying control signals to the transistors) and a signal-cable breakdown. Owing to the high speed, the (uncontrolled) induced voltage from the permanent magnets is higher than the DC-side voltage and the faulted in-wheel motor starts to regenerate power, which is diverted into the supercapacitor. A speed-dependent braking torque, shown in Figure 8(b), is thereby generated by the faulted in-wheel motor.

Figure 8 (a)-(c) PLECS simulation of an inverter shutdown: (a) power from battery and supercapacitor; (b) generated torque on the faulted in-wheel motor and (c) phase currents in the faulted in-wheel motor. (d)-(f) corresponding simulation of the vehicle dynamics: (d) longitudinal tyre forces; (e) lateral tyre forces and (f) vehicle steering angles

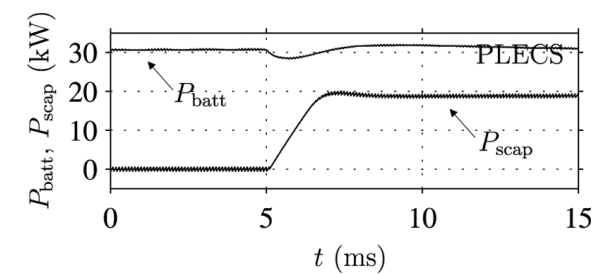

(a)

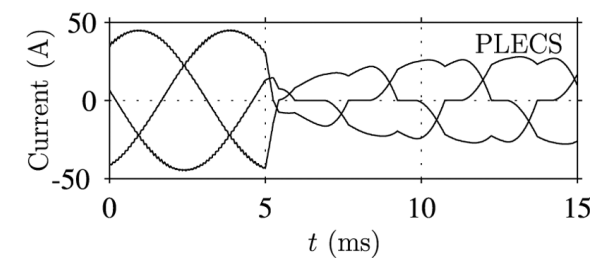

(c)

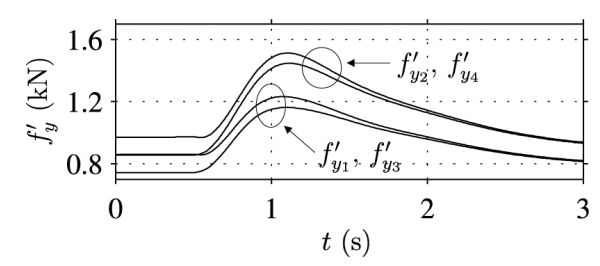

(e)

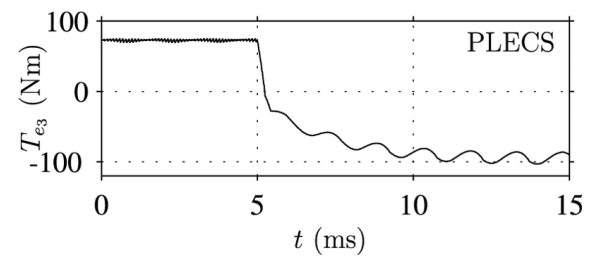

(b)

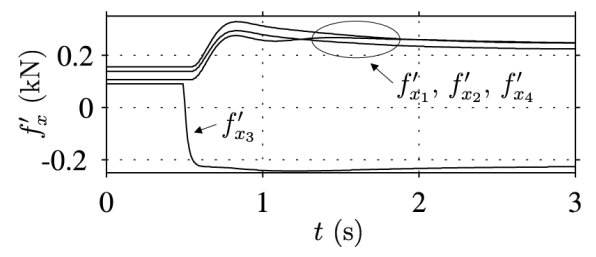

(d)

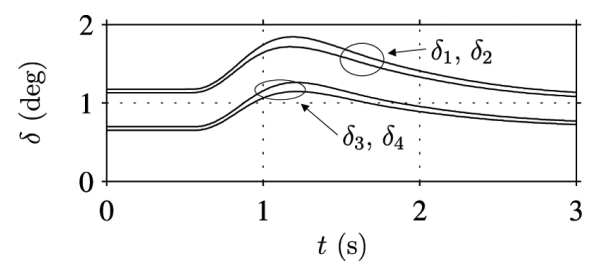

(f)

Results from a corresponding simulation of the vehicle dynamics are shown in Figures $8(\mathrm{~d})-(\mathrm{f})$. The vehicle is following a left-turn trajectory with a radius of $350 \mathrm{~m}$ and the inverter shutdown strikes the rear-left in-wheel motor at $t=0.5 \mathrm{~s}$. The vehicle trajectory (not shown) is virtually unaffected since the control architecture manages to rapidly re-distribute the tyre forces by changing the steering angles and increasing the torque from the remaining in-wheel motors (manifested by an increase in $f_{x_{1}}^{\prime}, f_{x_{2}}^{\prime}$ and $\left.f_{x_{4}}^{\prime}\right)$.

\subsection{Vehicle response to loss of power in an ACM}

A potentially more hazardous fault condition is evaluated in Figures 9 and 10 . The vehicle is following a left-turn curve trajectory with a radius of $350 \mathrm{~m}$ at the constant 
speed $v=25 \mathrm{~m} / \mathrm{s}(90 \mathrm{~km} / \mathrm{h})$. At $t=1 \mathrm{~s}$, the power to the rear-left ACM is lost. This is represented by a loss of propulsion power in the rear-left in-wheel motor and maintaining the steering angle fixed. The speed of the in-wheel motor is below the limit where the induced voltage in the faulted in-wheel motor starts to regenerate power into the DC side. The solid lines in Figures 9 and 10(a)-(c) correspond to the situation when no modification is made to the tyre-force allocation. As is clearly seen, the erroneous allocation quickly causes a large displacement in the vehicle trajectory, which cannot be compensated for by the path controller. The dashed lines in Figures 9 and 10(d)-(f), on the other hand, represent the situation when the constraints used in the tyre-force allocation are updated so that the tyre-force on the rear-left wheel is left uncontrolled (although known). With this modification, the remaining tyre forces are properly allocated and the vehicle trajectory is virtually unaffected, i.e., the fault condition is handled properly.

Figure 9 Resulting vehicle speeds and trajectory following a loss of power of the rear-left ACM. With (dashed lines) and without (solid lines) an adaptation of the actuator limitations used in the force-allocation process

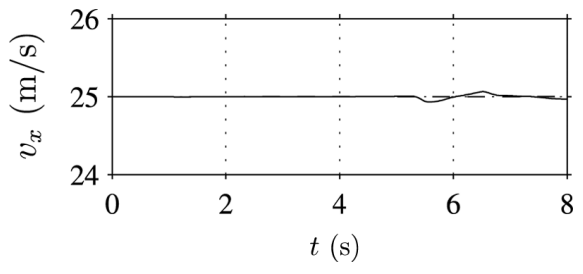

(a)

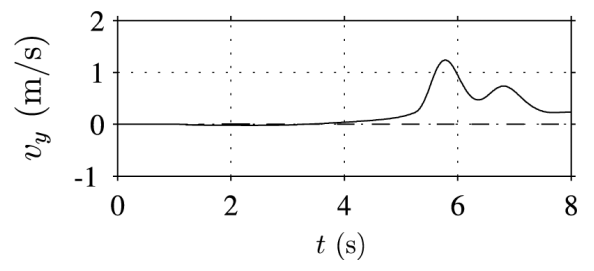

(b)

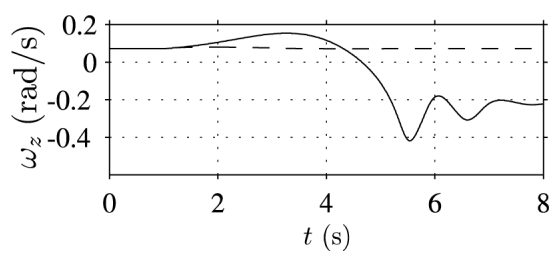

(c)

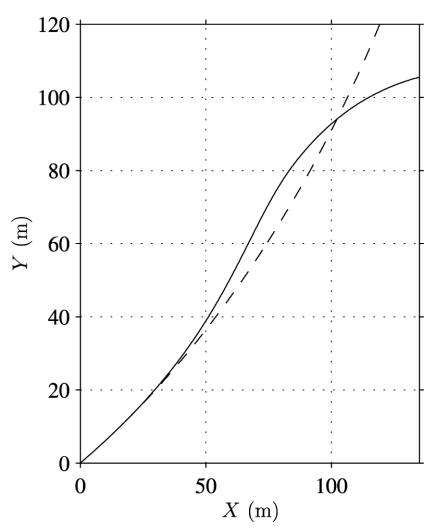

(d) 
Figure 10 Resulting steering angles, vehicle lateral force and rear-left lateral tyre force corresponding to Figure 9: (a)-(c): without adaptation and (d)-(f): with adaptation

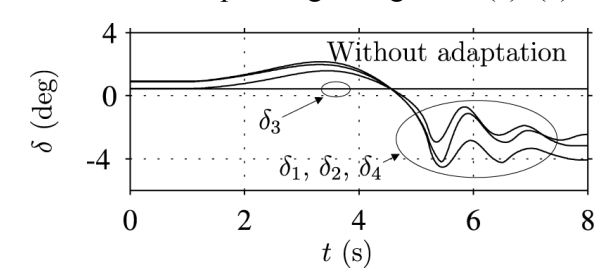

(a)

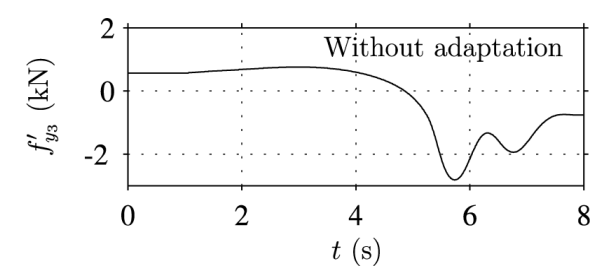

(c)

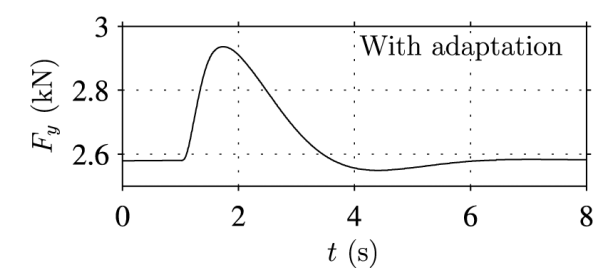

(e)

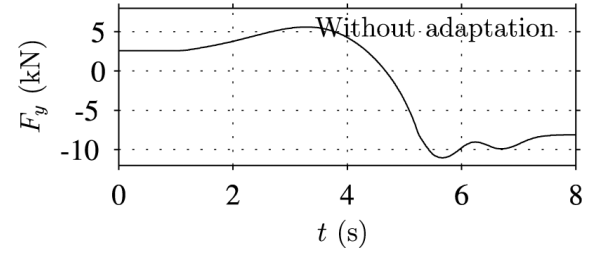

(b)

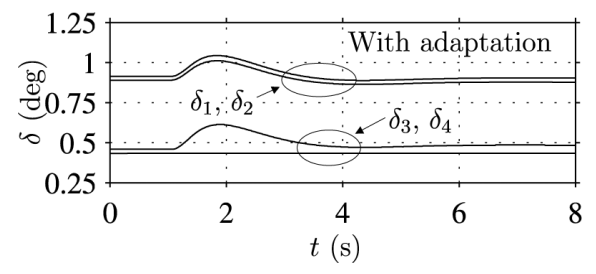

(d)

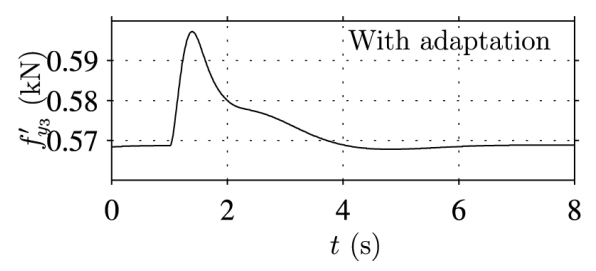

(f)

The difference between the results illustrated in Figures 8-10 shows that the control architecture, owing to the feedback control used in the path controller, generally is capable of maintaining vehicle stability, also during certain fault conditions. While this is not true during major faults and extreme driving situations, the situation can, however, be improved by incorporating knowledge of the present limitations arising in the faulted actuators.

\subsection{Unwanted interaction between actuators due to limitations in computational capacity}

The effect of a too limited computational capacity, manifested as slow sampling used in the optimisation solver, is demonstrated in Figure 11. The vehicle follows a straight path at the constant speed $v=33.3 \mathrm{~m} / \mathrm{s}(120 \mathrm{~km} / \mathrm{h})$. At $t=1 \mathrm{~s}$, the driver commands a sudden braking manoeuvre, manifested as a desired retardation of $3 \mathrm{~m} / \mathrm{s}^{2}$. Figure 11 shows the generated forces from the front-right in-wheel motor and the total longitudinal force on the front-left tyre (similar curves arise for all wheels). The solid lines represent a situation when the output of the optimisation solver is updated at $25 \mathrm{~Hz}$. Owing to the low sampling frequency, the generated braking force from the in-wheel motor arises too slowly which, in turn, causes the friction brake to activate. As can be seen, the unwanted interaction between the in-wheel motor and friction brake results in significant oscillation in the longitudinal corner force. The problem essentially vanishes when the optimisation 
solver is updated at a higher frequency, which is illustrated by the dashed lines in Figure 11 where a sampling frequency of $100 \mathrm{~Hz}$ is adopted.

Figure 11 Total longitudinal corner force $\left(f_{x_{2}}\right)$ and force from the friction brake $\left(T_{f} / r_{\mathrm{W}}\right)$ on the front-right wheel corner when the optimisation solver is updated at $25 \mathrm{~Hz}^{2}$ (solid lines) and $100 \mathrm{~Hz}$ (dashed lines), respectively

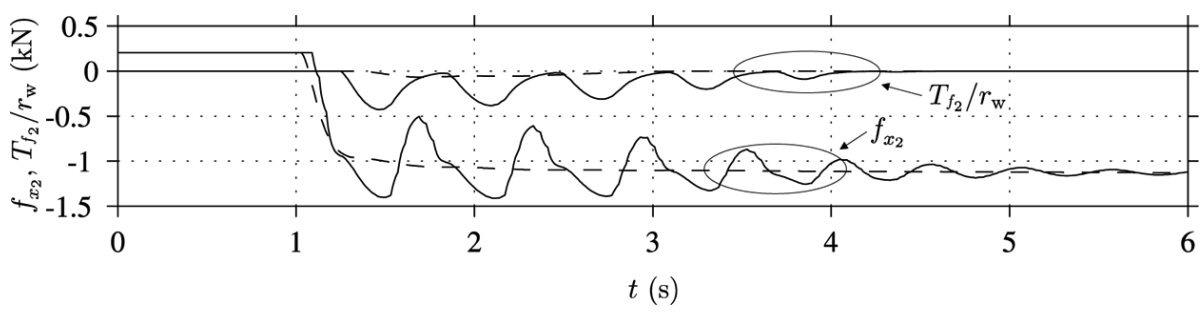

\section{Conclusion}

This paper has presented a simple method, suitable for on-board implementation, to allocate tyre forces in electric vehicles equipped with in-wheel motors and individual steering actuators. The control architecture considered was evaluated by introducing electric faults in a vehicle simulation model, which included the effects of non-ideal actuator dynamics and force constraints. The evaluation demonstrated that the control architecture's general ability to ensure vehicle stability is good. However, during major faults and extreme driving situations, the controller was not able to maintain vehicle stability unless the constraints used in the optimisation process used for tyre-force allocation were adapted to the specific fault condition. Finally, it was demonstrated how a limited computational capacity, manifested as a slow sampling used in the optimisation solver, combined with non-ideal actuators, can result in unwanted interactions between the individual actuators and affect vehicle stability.

From the above discussion, it is evident that methods for rapid detection and characterisation of faults that disable the actuators need to be further developed and incorporated into the control architecture. Further research should also be carried out to investigate to what extent the vehicle's path controller can be redesigned so that it can better suppress non-ideal effects in the actuator and tyre dynamics without the need for large modifications in the optimisation process used for force allocation.

\section{Acknowledgements}

This work was financed in part by the Swedish National Energy Agency. The authors are grateful to Mr. Jens Groot at Volvo Technology Corporation for supplying the lithium-ion battery model. 


\section{References}

Andreasson, J. and Bünte, T. (2006) 'Global chassis control based on inverse vehicle dynamics models', Supplement to Vehicle System Dynamics, Vol. 44, pp.321-328.

Andreasson, J., Laine, L. and Fredriksson, J. (2004) 'Evaluation of a generic vehicle motion control architecture', Proc. FISITA World Automotive Conference, Barcelona, Spain, 23-27 May, Paper: F2004F360.

Bose, B.K. (2002) Modern Power Electronics and AC Drives, Prentice-Hall, Upper Saddle River, NJ, USA.

Capel, A. (2001) 'Mathematical model for the representation of the electrical behaviour of a lithium cell', Proc. 32nd Power Electronics Specialist's Conference, Vancouver, Canada, Vol. 4, 17-21 June, pp.1976-1981.

Chan, C.C. and Wong, Y.S. (2004) 'The state of the art of electric vehicles technology', Proc. 4th International Conference on Power Electronics and Motion Control, Xi'an, China, 14-16 August, Vol. 1, pp.46-57.

Dietrich, P., Laurent, D., Paganelli, G., Tsukada, A., Walser, D., Magne, P-A., Büchi, F.N., Varenne, P., Kötz, R., Freunberger, S., Delfino, A. and Olsommer, D. (2005) 'Concept, design and first results of the light-weight-fuel-cell-vehicle 'Hy-Light', Proc. 21st International Battery, Hybrid and Fuel Cell Electric Vehicle Symposium and Exposition, Monaco, 2-6 April, On a CD-rom, pp.1-8.

Emadi, A., Williamson, S.S. and Khaligh, A. (2006) 'Power electronics intensive solutions for advanced electric, hybrid electric, and fuel cell vehicular power systems', IEEE Transactions on Power Electronics, Vol. 21, No. 3, pp.567-577.

Gao, L., Liu, S. and Dougal, R.A. (2002) 'Dynamic lithium-ion battery model for system simulation', IEEE Transactions on Components and Packaging Technologies, Vol. 25, No. 3, pp.495-505.

Jahns, T.M. and Caliskan, V. (1999) 'Uncontrolled generator operation of interior PM synchronous machines following high-speed inverter shutdown', IEEE Transactions on Industrial Applications, Vol. 35, No. 6, pp.1347-1357.

Jonasson, M. and Wallmark, O. (2006) 'Stability of an electric vehicle with permanent magnet inwheel motors during electrical faults', Proc. 22nd International Battery, Hybrid and Fuel Cell Electric Vehicle Symposium and Exposition, CD-ROM, Yokohama, Japan, 23-28 October, pp.1248-1259.

Jonasson, M., Zetterström, S. and Trigell, A.S. (2006) 'Autonomous corner modules as an enabler for new vehicle chassis solutions', Proc. FISITA World Automotive Conference, Yokohama, Japan, 22-27 October, Paper: F2006V054T.

Knobel, C., Pruckner, A. and Bünte, T. (2006) 'Optimized force allocation - a general approach to control and to investigate the motion of over-actuated vehicles', Mechatronic Systems, Vol. 4, Part 1.

Orend, R. (2005) Proc. 16th IFAC World Congress, Prague, Czech Republic, 4-8 July.

Pacejka, H.B. (2002) Tyre and Vehicle Dynamics, Butterworth-Heinemann, Oxford, UK.

Siemens, V.D.O. (2006) Car Motors will Disappear into the Wheels: Siemens VDO Starts eCorner Development, WWW-pages, Source: Internet: <URL:http://www.siemensvdo.com>, 21.09.2006.

Valášek, M. (2003) 'Design and control of under-actuated and over-actuated mechanical systems - challenges of mechanics and mechatronics', Vehicle Systems Dynamics, Vol. 40, Supp., pp.37-50. 
Wallmark, O. (2006) Control of Permanent-Magnet Synchronous Machines in Automotive Applications, PhD Dissertation, Chalmers University of Technology, Göteborg, Sweden.

Zetterström, S. (2002) 'Electromechanical steering, suspension, drive and brake modules', Proc. 56th IEEE Vehicular Technology Conference, Vancouver, Canada, Vol. 3, 24-28 September, pp.1856-1863.

\section{Notes}

${ }^{1}$ For an introduction on power electronics and electric drives, see, e.g., Bose (2002).

${ }^{2}$ MATLAB and Simulink are registered trademarks of The Mathworks, Inc., Natick, Massachusetts, USA.

${ }^{3}$ PLECS is a registered trademark of Plexim GmbH, Zürich, Switzerland.

\section{Appendix}

Table 1 Nomenclature

\begin{tabular}{ll}
\hline Symbol & Abbreviation \\
\hline$\alpha_{i}$ & Side-slip angle of $i$ th tyre \\
$\beta$ & Vehicle slip angle \\
$\delta_{i}$ & Steering angle of $i$ th tyre \\
$\mu_{i}$ & Friction coefficient of $i$ th tyre \\
$w_{\mathrm{w}}$ & Rotational speed of $i$ th wheel \\
$w_{z}$ & Vehicle yaw speed \\
$\rho$ & Path curvature \\
$\mathbf{f}=\left[f_{x 1} f_{y 1} \cdots f_{x 4} f_{y 4}\right]^{T}$ & Vehicle corner forces \\
$\mathbf{f}^{\prime}=\left[f_{x_{1}}^{\prime} f_{y_{1}}^{\prime} \cdots f_{x_{4}}^{\prime} f_{y_{4}}^{\prime}\right]^{T}$ & Tyre forces \\
$\mathbf{f}_{\text {glob }}=\left[F_{x} F_{y} M_{z}\right]^{T}$ & Vehicle global forces \\
$f_{i}^{\max }$ & Maximum tyre force of $i$ th tyre \\
$f_{z_{i}}$ & Normal load of $i$ th tyre \\
$P_{\text {batt }}$ & Power delivered by battery \\
$P_{\text {scap }}$ & Power absorbed by supercapacitor \\
$T_{e_{i}}$ & In-wheel motor torque on $i$ th wheel \\
$T_{f_{i}}$ & Torque from $i$ th friction brake \\
$T_{i}$ & Total torque on $i$ th wheel \\
$V$ & Vehicle speed \\
$v_{x}$ & Longitudinal vehicle speed \\
$v_{y}$ & Lateral vehicle speed \\
\hline & \\
\hline
\end{tabular}


Table 1 Nomenclature (continued)

\begin{tabular}{ll}
\hline Sub and superscripts & Abbreviations \\
\hline, & Tyre coordinate system \\
$I$ & Tyre number \\
$J$ & Line number, force constraints \\
\hline
\end{tabular}

Table 2 Vehicle parameters

\begin{tabular}{lll}
\hline Parameter & Symbol & Value \\
\hline Vehicle mass & $m$ & $1400 \mathrm{~kg}$ \\
Vehicle dimensions & $\left\{d_{1}, d_{2}, d_{3}, d_{4}\right\}$ & $\{0.7,0.7,1.1,1.3\} \mathrm{m}$ \\
Height to centre of gravity & & $0.42 \mathrm{~m}$ \\
Roll, pitch and yaw inertia & $\left\{I_{x}, I_{y}, I_{z}\right\}$ & $\{500,1700,1800\} \mathrm{kg} \mathrm{m}{ }^{2}$ \\
Spring rates & $\left\{k_{1}, k_{2}, k_{3}, k_{4}\right\}$ & $\{25,25,30,30\} \mathrm{kN} / \mathrm{m}$ \\
Damper rates & $\left\{c_{1}, c_{2}, c_{3}, c_{4}\right\}$ & $\{1.5,1.5,1.5,1.5\} \mathrm{kNs} / \mathrm{m}$ \\
Anti-roll bar stiffness & & $15 \mathrm{kNm} / \mathrm{rad}$ \\
Wheels, tyres and brakes & & \\
Nominal wheel load & $f_{z}$,nom & $3300 \mathrm{~N}$ \\
Wheel radius & $r_{\mathrm{w}}$ & $0.32 \mathrm{~m}$ \\
Tyre dimensions & & $225 / 45 \mathrm{R} 17$ \\
Maximum friction brake force & lo & $2580 \mathrm{~N}$ \\
Minimum friction brake force & up & $0 \mathrm{~N}$ \\
\hline
\end{tabular}

